\title{
EUSO: using high energy cosmic rays and neutrinos as messengers from the unknown universe (*)
}

\author{
Livio Scarsi \\ Istituto di Astrofisica Spaziale e Fisica Cosmica (IASF), CNR, Sezione di Palermo, Italy
}

\begin{abstract}
Exploiting the earth atmosphere as a giant detector for the incoming extraterrestrial flux of high energy cosmic rays and cosmic neutrinos, the mission «EUSO-Extreme Universe Space Observatory» is devoted to the exploration of the domain of the highest energy processes occurring in the universe up to its accessible boundaries. The observable is provided by the air nitrogen fluorescence light emitted in the UV band 300-400 $\mathrm{nm}$ by the extensive air showers produced by the cascading processes of the primary cosmic radiation particles interacting with the atmosphere. The EUSO telescope is based on a double Fresnel lens optics (diameter $2.5 \mathrm{~m}$ ) coupled to a highly pixelized focal surface composed multianode PMTs; the image at the earth surface is detailed at $1 \mathrm{~km}^{2}$ over a total of several hundred thousand of $\mathrm{km}^{2}$. EUSO will fly on the International Space Station accommodated as external payload of the European Space Agency Columbus module. The mission is scheduled to last 3 years, with the start of operations foreseen for 2007/8. The expectations are of a collection rate of a thousand events/year for cosmic rays at $E>10^{20} \mathrm{eV}$ together with tens/hundreds of cosmic neutrinos at energy above about $4 \times 10^{19} \mathrm{eV}$. EUSO is the result of the collaborative effort of several institutions in Europe, Japan and USA and it is conceived within the science program sponsored by various space agencies coordinated by ESA.
\end{abstract}

Key words fluorescence - cosmic rays - neutrinos extensive air showers

\section{Introduction}

Cosmic radiation can be considered the «particle channel» complementing the «electromagnetic channel» proper of conventional astronomy.

Mailing address: Prof. Livio Scarsi, Istituto di Astrofisica Spaziale e Fisica Cosmica (IASF), CNR, Sezione di Palermo, Via U. La Malfa 153, 90146 Palermo, Italy; e-mail: livio. scarsi@pa.iasf.cnr.it

(*) This text is largely derived from contributions made by the author in 2000/2001 to other Workshops and Conferences and from documentation submitted by the EUSO Consortium to the European Space Agency as a part of the EUSO proposal.
A classic presentation of the cosmic ray energy spectrum is shown in fig. 1; an unconventional view (which I borrowed from a colleague of Karlsruhe, where it was first shown at the ceremonial organized in honor of Dr. Shatz) is given in fig. 2 to illustrate in an anthropomorphic perspective the features conventionally called «knee» (around $10^{15} \mathrm{eV}$ ) and «ankle» (above 5 $\left.\times 10^{18} \mathrm{eV}\right)$. The remarkable «feminine leg» in the figure is that of the famous German movie star Marlene Dietrich.

Today substantial progress has been made in the knowledge of the nature of cosmic rays of the relatively modest energies (up to the «knee» at $10^{14}-10^{15} \mathrm{eV}$ ). On the other hand the cosmic radiation on the higher energy side presents us with the challenge of understanding its origin and its connection with fundamental problems in cosmology and astroparticle physics. 


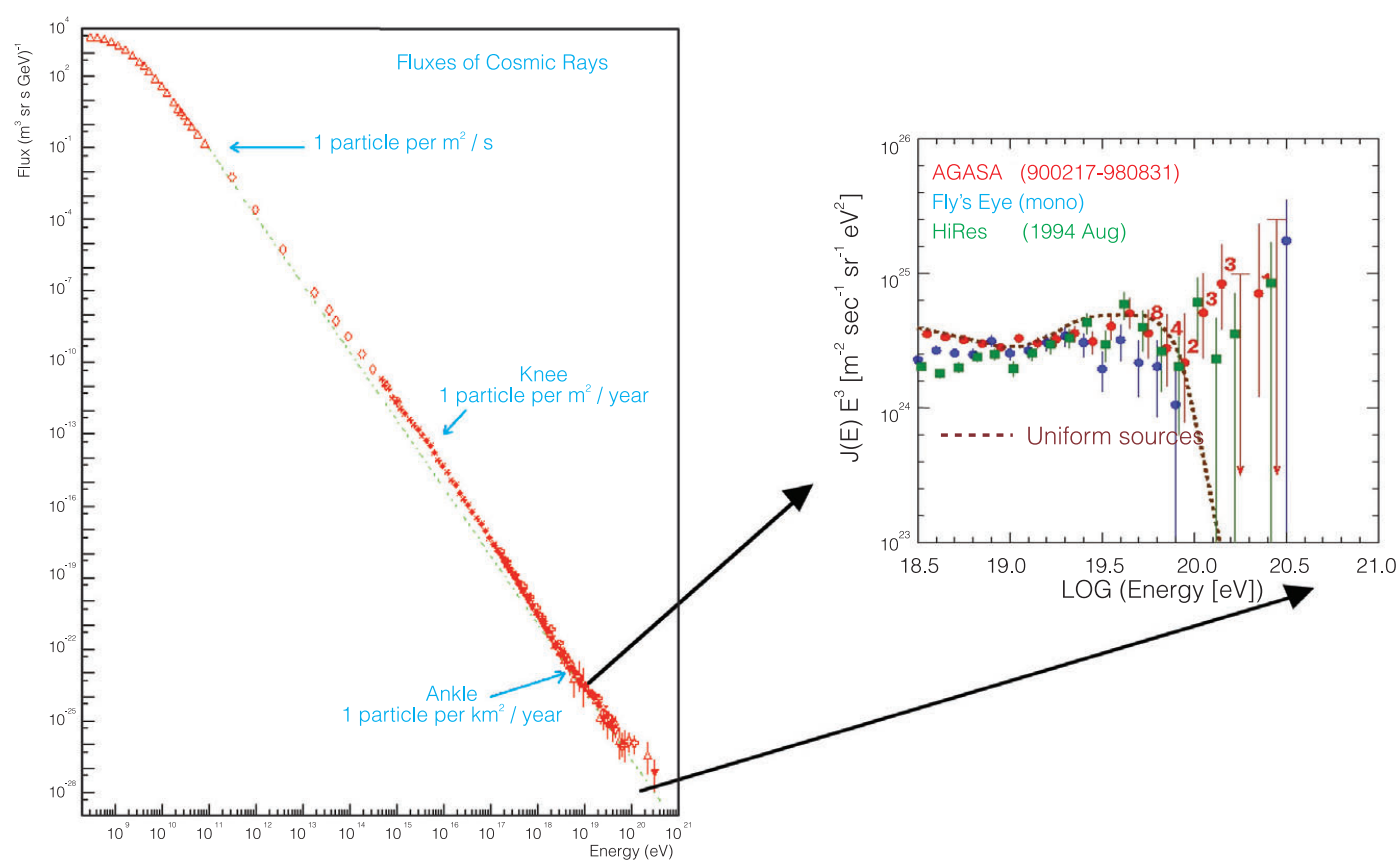

Fig. 1. The observed cosmic ray spectrum for $E>10^{8} \mathrm{eV}$ showing the principle features. The inset shows the high-energy part with the overall E-3 dependence removed as observed by AGASA (Takeda et al., 1998), Fly's Eye and HiRes. The dashed line shows the effect of the GZK cut-off assuming a homogenous source population filling the universe. The numbers are the actual number of events in each bin.

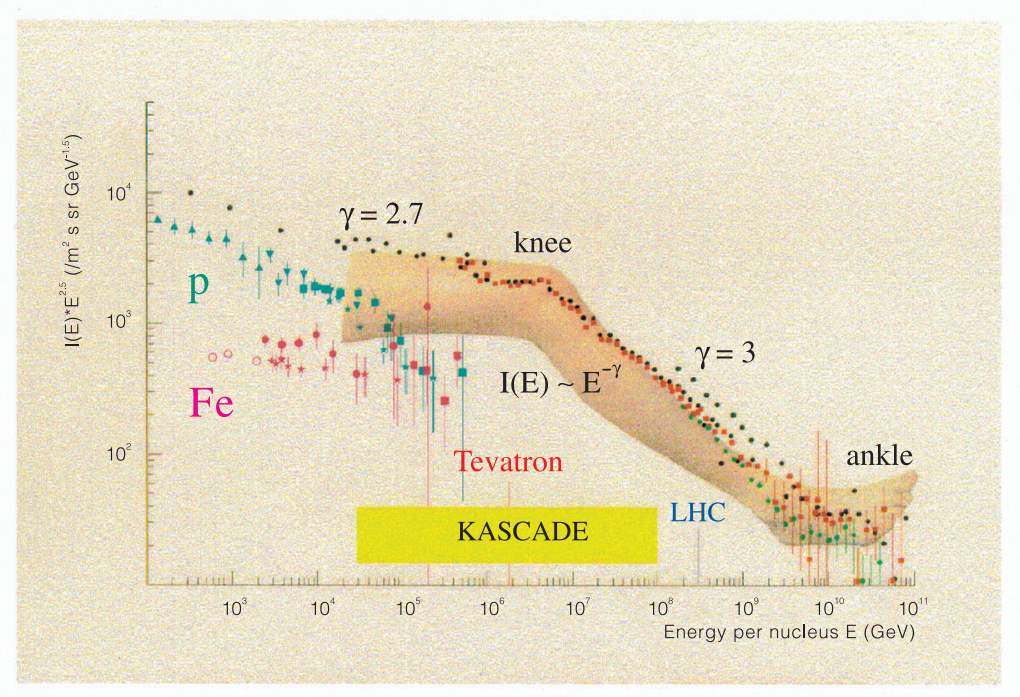

Fig. 2. The cosmic ray energy spectrum: anthropomorphic presentation. 
Focal points are represented by:

i) The change in the spectral index at $\sim 5 \times$ $\times 10^{18} \mathrm{eV}($ (ankle»).

ii) Existence of «cosmic rays» with energy $E>10^{20} \mathrm{eV}$ : (EECR) (fig. 1).

A direct question arising is: what is the maximum cosmic ray energy, if there is any limit? Addressing the theoretical issue concerning the production and propagation of $10^{20} \mathrm{eV}$ primary quanta is problematic and involves processes still little known.

\section{The universe and the probing depth of the extreme energy cosmic radiation}

From the document «Report on the Accommodation of EUSO on the Columbus Exposed Payload Facility: ESA/MSM-GU/2000, 462/AP/ RDA, December 2000».

Soon after the discovery of the Cosmic Microwave Background radiation (CMB) by Penzias and Wilson in 1965, Greisen, and, independently, Zatsepin and Kuzmin pointed out that this radiation would make the universe opaque to cosmic rays of sufficiently high energy. For protons, e.g., this occurs when the pion production threshold is reached (about $5 \times 10^{19} \mathrm{eV}$, if the Lorentz transformations of Relativity still hold at $\left.\gamma \geq 10^{11}\right)$. The reaction $p+\gamma \rightarrow \Delta^{+} \rightarrow p+\pi^{\circ}$ or $n+\pi^{+}$will lead to an effective attenuation length of $50 \mathrm{Mpc}$ for a proton of $10^{20} \mathrm{eV}$. This is about the size of the Virgo cluster to which our galaxy belongs, and is just a small fraction of the size of the universe. Table I summarizes the effects introduced on the primary EECR components, including gamma rays, by the interaction with the CMB. To a much lesser extent, neutrinos decay above $10^{21}-10^{22} \mathrm{eV}$ by $Z_{0}$-resonance with Cosmic Neutrino Background (CNB).

The Greisen-Zatsepin-Kuzmin effect shapes in a complicated way the form of the observed energy spectrum of the EECR as a function of the distribution of the extragalactic sources in the universe (see fig. 3a,b).

\subsection{Sources of extreme energy cosmic rays}

Many sources of high-energy particles could exist in the universe at distances beyond the GZK length of $50 \mathrm{Mpc}$. The predicted sharp cut-off of energy spectrum above $5 \times 10^{19} \mathrm{eV}$ may not be present if a possible high abundance of cosmological neutrino events, or others from nearby sources, are dominant. A significant bump should exist at $10^{19}-10^{20} \mathrm{eV}$ due to the GZK effect for protons, because cosmological protons that were accelerated to the super-GZK energies decay down to the trans-GZK energies, and pile up at sub-GZK energies. The details of the energy spectrum in the trans-GZK energy regime $\left(10^{19}-2 \times 10^{20} \mathrm{eV}\right)$ depend on the model of the evolution of the universe from about 10 billion years ago (or $z \sim 5$ ). High event statistics can provide information on the evolution of the highest energy universe. This information on the evolution of universe may be distorted by the probable existence of super-GZK neutrinos. However, using EUSO the neutrino energy spectrum can be identified and directly measured, and the non-neutrino spectrum can be statistically corrected by subtracting relevant neutrinos and their

Table I. Extreme energy processes that cut-off the energy spectrum of particles in universe.

\begin{tabular}{llll}
\hline \hline & \multicolumn{1}{c}{ Process } & \multicolumn{1}{c}{ Cut-off energy } & Mean free path \\
\hline Protons & $p+\gamma_{2.7 \mathrm{~K}} \rightarrow \pi^{\circ}+X$ & $\mathrm{eV}$ & $50 \mathrm{Mpc}$ \\
Nuclei & $A+\gamma_{2.7 \mathrm{~K}} \rightarrow \Delta^{++}+X$ & $\geq 5 \times 10^{18} \mathrm{eV} / \mathrm{n}$ & $100 \mathrm{Mpc}$ \\
Gamma-rays & $\gamma+\gamma_{2.7 \mathrm{~K}}$ & $\geq 10^{14} \mathrm{eV}\left(\right.$ at $\left.10^{20} \mathrm{eV}\right)$ & $10 \mathrm{Mpc}\left(\right.$ at $\left.10^{20} \mathrm{eV}\right)$ \\
Neutrinos & $v+v_{1.95 \mathrm{~K}} \rightarrow\left(W / Z_{0}\right)+X$ & $\geq 4 \times 10^{22} \mathrm{eV}$ & $40 \mathrm{Gpc}$ \\
\hline
\end{tabular}



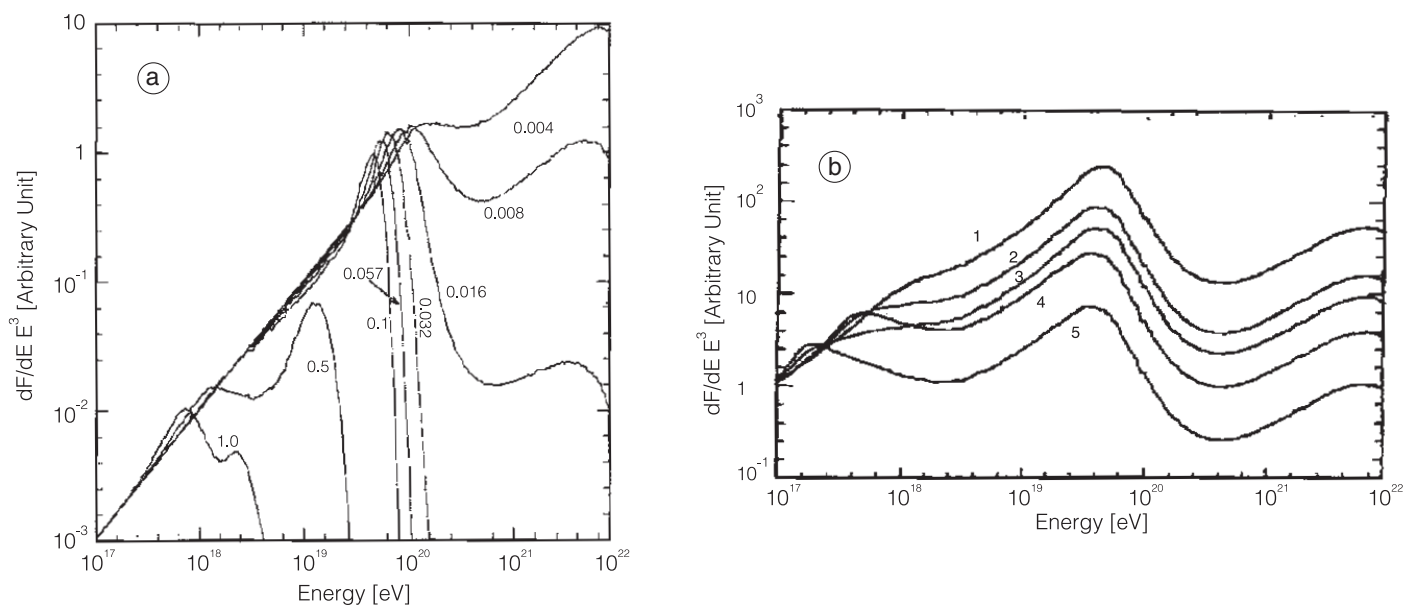

Fig. 3a,b. a) Energy spectra from a single source of protons with an $E^{-2}$ spectrum, for various source distances between $z=0: 004$ and 1 (i.e. between 2 and $5000 \mathrm{Mpc}$ ). b) The nucleon spectrum at the trans-GZK and superGZK energies predicted from different models of the cosmic evolution, including the hypothetical component at EE up to $10^{23} \mathrm{eV}$. Curve 1 is for the case of homogeneous evolution for the redshifts $0 \leq z \leq 2$. Curve 5 shows non-homogeneous distribution up to $z=4$ in which early cosmological era has more active EE sources. Curves 2-4 are for intermediate models. Cosmological neutrinos and their secondaries are not included in this figure.

secondaries. The evolution of energetic sources in the universe will be examined by such statistical means.

The current summary of the data from AGASA, Fly's Eye and HiRes is shown in the insert of fig. 1 and suggests that the observed cosmic ray spectrum is not necessarily cut-off following the predicted GZK process for protons and nuclei. It suggests that either the majority of cosmic rays originated within the GZK length $(<50 \mathrm{Mpc})$, or the relativity principle fails at extreme energies, or some other unknown sources exists.

Concerning the distribution of arrival directions, AGASA and worldwide data summary of cosmic rays above about the GZK cut-off energy show a quasi-isotropic distribution in the sky clearly suggesting an extragalactic origin. Among them, 6 pairs and 1 triple set of spatially correlated events within 2 years from 58 events were recognized by AGASA only, while the world-wide data show 9 pairs and two triples. The chance coincidence probability for these «clusters» of events is less than $0.07 \%$, and therefore, the particles of a «cluster» possibly had the same sources.
Although the existence of the highest energy cosmic rays is proven, their origin is still an enigma despite the efforts of many theorists and experimentalists. One is led to the conclusion that they have an entirely different origin from the lower energy cosmic rays. The present data raise questions of great importance for astrophysics, cosmology, and fundamental physics.

Focusing the attention on the primary sources, the general production mechanisms proposed for the EECRs can be classified as:

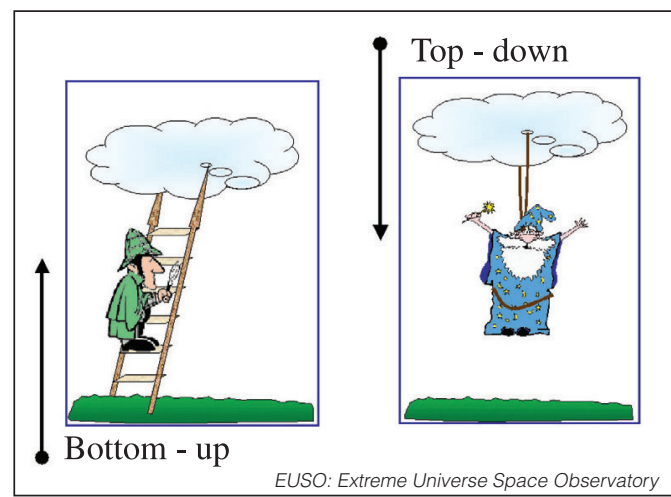


- Bottom-up, with acceleration in rapidly evolving processes occurring in astrophysical objects. The scenario involves astrophysical objects such as, e.g., AGNs and AGN radio lobes. The study of these objects is, besides radio observations, a main goal of x-ray and gammaray astrophysics of the late 90's. An extreme case in this class is represented by the gamma ray bursts, found to be located at cosmological distances. The observation of "direction of arrival and time» coincidences of GRBs and extreme energy neutrinos $\left(E \geq 10^{19} \mathrm{eV}\right)$ in the EUSO mission could provide a crucial test for the identification of the observed GRBs as EECR sources in spite of their location at distances well above the GZK limit.

- Top-down processes. This scenario arises from the cascading of ultrahigh energy particles from the decay of topological defects. Cosmic strings would play an essential role for releasing the $\mathrm{x}$-bosons emitting the highest energy quarks and leptons. This process could occur in the nearby universe. The relics of an early inflationary phase in the history of the universe may survive to the present as a part of dark matter and account for those unidentified EECR sources active within the GZK boundary limit. Their decays can give rise to the highest energy cosmic rays, either by emission of hadrons and photons, or through production of EE neutrinos.

From the astroparticle physics point of view, the EECRs have energies only a few decades below the grand unification energy $\left(10^{24}-10^{25} \mathrm{eV}\right)$, although still far from the Plank mass of $10^{28} \mathrm{eV}$.

\subsection{Neutrino induced air showers}

Neutrinos with high enough energy can produce detectable EAS observable by EUSO. This will provide valuable information on their origin together with that of the EECR. Not suffering the GZK effect and being immune from magnetic field deflections, or from a delay caused by the quantum relativity effects, neutrinos are ideal for disentangling source-related mechanisms from propagation-related effects. The opening of the neutrino astronomy channel will allow the extreme boundaries of the universe to be probed.

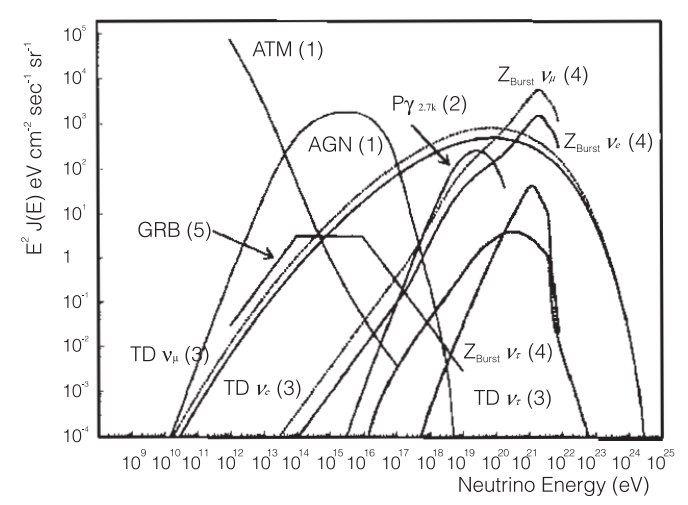

Fig. 4. Predicted neutrino fluxes for various models. The large range of predicted fluxes should be noted as should the number of models which exceeds the number of events with energies $>10^{20} \mathrm{eV}$ ! (1) Stecker and Salamon (1996); (2) Stecker et al. (1991); (3) Sigl et al. (1999), $m_{X}=10^{16} \mathrm{GeV}, X->q+q$, supersymmetric fragmentation; (4) Yoshida et al. (1998), $m_{v}=1 \mathrm{eV}$, primary $\Phi_{v} \sim E^{-1}$; (5) Waxmann and Bachall (1997).

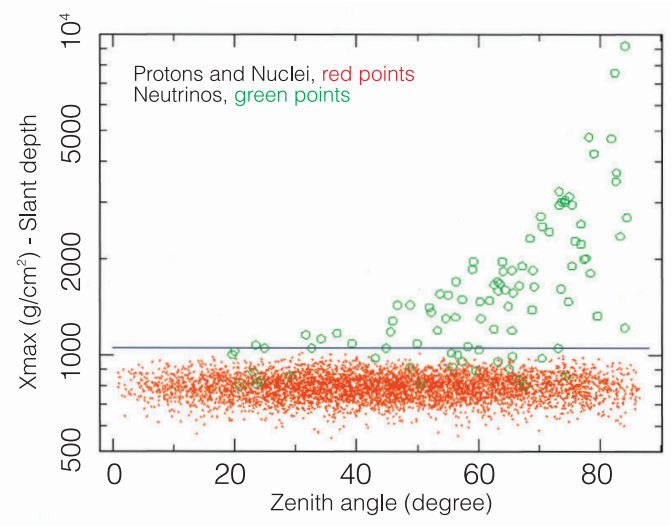

Fig. 5. Shower depth distribution from Monte Carlo simulations showing how neutrino and proton and nuclei induced events can be distinguished.

However, neutrinos are elusive objects with a low interaction probability, to such an extent that they can be neglected as observable EAS initiators for all ground based detectors, present or planned. Even for the largest planned ground based cosmic ray detector (the Auger project), 
in the most optimistic case, the expected rate is only a few events per year. EUSO, with its large sensitive area and accessible mass target of the order of $10^{13} \mathrm{t}$ of atmosphere, will be sensitive to this class of events.

The expected neutrino event rate ranges from a few events per year (GZK processes, AGN, GRB sources, etc.) to 150 per year according to the effectiveness of the «topological defects» hypothesis (see fig. 4). Observationally, neutrinoinduced EAS can be distinguished from background events and from other EECR EAS by selecting events with large zenith angles which initiate deep in the atmosphere (fig. 5). A nearly horizontal $\tau$-neutrino event with an energy $>10^{19} \mathrm{eV}$ can be identified by a «double bang» structure. Both the initial shower in the $v_{\tau} \rightarrow \tau$ interaction, and another, by the $\tau$-decay, can be seen because of the long path length $(\sim 1000$ $\left.\left[E / 10^{20} \mathrm{eV}\right] \mathrm{km}\right)$ for $\tau$-decays.

\section{Observational problems}

The extremely low value for the EECR flux, corresponding to about 1 event per $\mathrm{km}^{2}$ and century at $E>10^{20} \mathrm{eV}$, and the extremely low value for the interaction cross section of neutrinos, make these components difficult to observe if not by using a detector with exceptionally high values for the effective area and target mass. The integrated exposure $\left(\sim 2 \times 10^{3} \mathrm{~km}^{2} \mathrm{yr} \mathrm{sr}\right)$ available today for the ground based arrays operational all over the world is sufficient only to show the «ankle» feature at $\sim 5 \times 10^{18} \mathrm{eV}$ in the cosmic ray energy spectrum and the existence of about ten events exceeding $10^{20} \mathrm{eV}$; the limited statistics excludes the possibility of observing significant structures in the energy spectrum at higher energies. Experiments carried out by means of the new generation ground-based observatories, HiRes (fluorescence) and Auger (hybrid), will still be limited by practical difficulties connected to a relatively small collecting area $\left(<10^{4} \mathrm{~km}^{2} \mathrm{sr}\right)$ and by a modest target mass value for neutrino detection.

To overcome these difficulties, a solution is provided by observing from space (fig. 6) the atmosphere UV fluorescence induced by the incoming extraterrestrial radiation, which exploits up to millions $\mathrm{km}^{2} \mathrm{sr}$ for the acceptance area and up to $10^{13} \mathrm{t}$ as target for neutrino interaction. This is the philosophy of the «AirWatch Programme» and «EUSO» is a space mission developed in the AirWatch framework.

The earth atmosphere in fact constitutes the ideal detector for the extreme energy cosmic rays and the companion cosmic neutrinos. The EECR particles, interacting with the air nuclei, give rise to propagating Extensive Air Showers (EAS) accompanied by the isotropic emission of ultraviolet fluorescence $(300-400 \mathrm{~nm})$ induced in nitrogen by the secondary charged particles in the EAS as a result of a complex relativistic cascade process; an isotropically diffuse opticalUV signal is also emitted following the impact on clouds, land or sea of the Cherenkov beam accompanying the EAS. A shower corresponding to a primary with $E>10^{19} \mathrm{eV}$ forms a significant streak of fluorescence light over 10-100 km along its passage in the atmosphere, depending on the nature of the primary, and on the pitch angle with the vertical.

Observation of this fluorescence light with a detector at distance from the shower axis is the best way to control the cascade profile of the EAS. When viewed continuously, the object moves on a straight path with the speed of light. The resulting picture of the event seen by the detector looks like a narrow track in which the recorded amount of light is proportional to the shower size at the various penetration depths in the atmosphere. From a Low Earth Orbit (LEO) space platform, the UV fluorescence induced in atmospheric nitrogen by the incoming radiation can be monitored and studied. Other phenomena such as meteors, space debris, lightning, atmospheric flashes, can also be observed, while the luminescence coming from the EAS produced by the cosmic ray quanta can be disentangled from the general background exploiting its fast timing characteristic feature.

EUSO observes at Nadir from an orbital height of about $400 \mathrm{~km}$. It is equipped with a wide angle Fresnel optics telescope $\left(60^{\circ}\right.$ full FoV) and the focal plane segmentation corresponding to about $1 \mathrm{~km}^{2}$ pixel size on the earth surface. The area covered on Earth is of about $160000 \mathrm{~km}^{2}$. Exploiting the high speed of the focal plane de- 


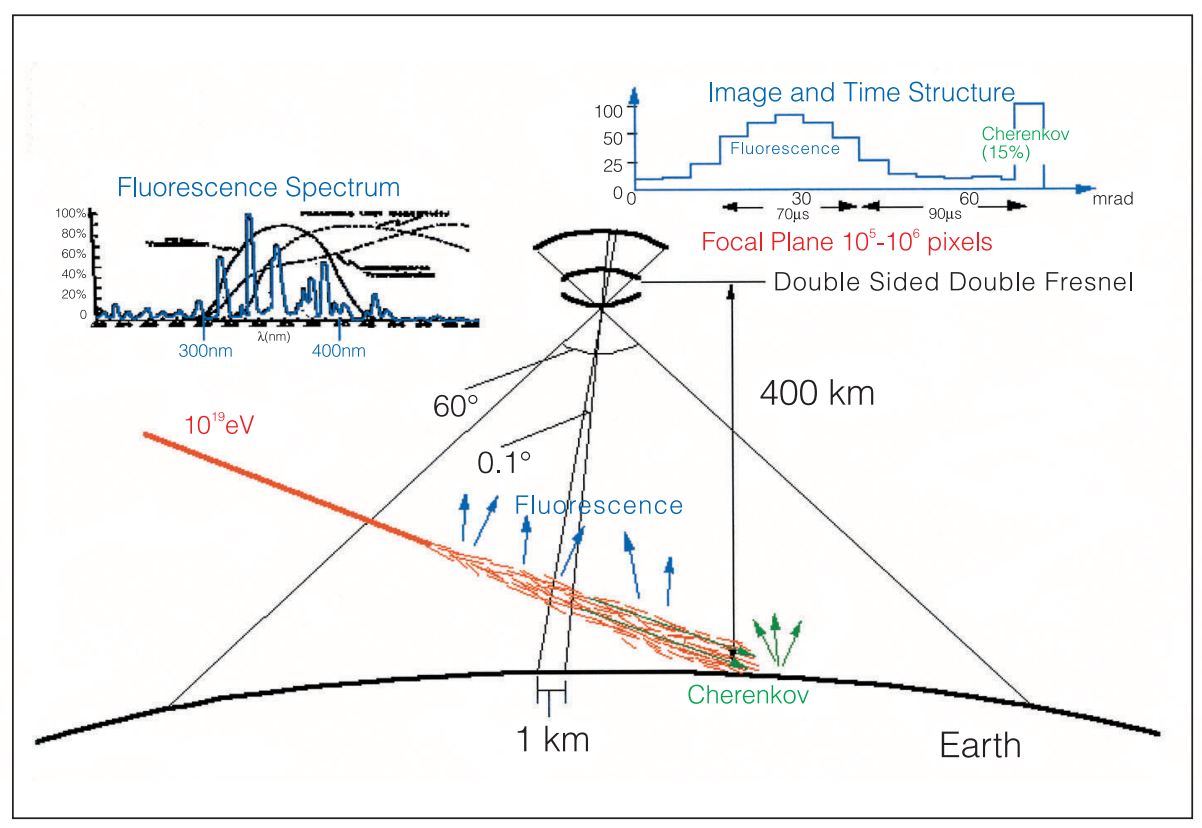

Fig. 6. Observation of EAS from space.

tector (10 ns class), EUSO is able to reconstruct the inclination of the shower track by the speed of progression of the projected image on the focal surface and to provide the tri-dimensional reconstruction of the EAS axis with a precision of a degree (or better) depending on the inclination. By measuring the EAS front luminosity with the PhotoElectrons (PE) detected by the MAPTs covering the focal surface, EUSO registers the longitudinal development of the EAS.

\subsection{EUSO general requirements and main goals}

For a significant observation from a space mission the assumed values are:

a) Geometrical exposure of $\left(5 \times 10^{4}-10^{5}\right) \mathrm{km}^{2}$ sr considering a duty cycle of $0.1-0.15$.

b) EAS energy threshold at about $5 \times 10^{19} \mathrm{eV}$.

EECR statistics - About $10^{3}$ events/year (an order of magnitude above those expected by the presently planned ground based experiments) to allow a quantitative energy spectral definition above $10^{20} \mathrm{eV}$, together with the evidence of possible anisotropy effects and clustering (if any) for the directions of arrival.

Neutrino events - The expected event rate ranges from several events/year (AGN, GRB source) to several events/day according to the effectiveness of the «topological defects» hypothesis. From the observational point of view, the neutrino induced EAS can be distinguished from background and from other EECR EAS by triggering on horizontal showers initiating deep inside the atmosphere. Moreover neutrinos with energy of about $10^{15}-10^{16} \mathrm{eV}$ interacting in the solid earth and emerging upward in the atmosphere create showers which can be detected by EUSO by means of the Cherenkov beamed signal induced in the atmosphere, extending the capability of EUSO to this lower neutrino astronomy energy band. A horizontal $\tau$-neutrino event at energies greater than $10^{19} \mathrm{eV}$ can be identified by a «double bang» structure. Both the initial shower at the $v_{\tau} \rightarrow \tau$ interaction, and 
another, by the $\tau$-decay, can be seen because of the long enough path-length $\left(\sim 1000\left[E / 10^{20}\right.\right.$ $\mathrm{eV}] \mathrm{km}$ ) for $\tau$-decays observable by EUSO. $\tau$-neutrinos above $10^{15} \mathrm{eV}$, on the other hand, will be observed and identified as earth-penetrating «upward» showers (by Cherenkov). High $v_{\tau}$ flux by the $v_{\mu} \rightarrow v_{\tau}$ oscillation and the low detection threshold energy for them allow EUSO to make oscillation experiments in space as well as $v_{\tau}$ astrophysics of AGN above $10^{15} \mathrm{eV}$.

\subsection{EUSO schematic outline}

EUSO, originally proposed to ESA in January 2000 for a free-flyer LEO mission, was approved in March 2000 for an «accommodation study» on the ISS International Space Station (ESA/ESTEC, 2000; Scarsi et al., 2000). As a result, EUSO is now undergoing a «bridging phase» to enter «phase A design» carried out by industry under an ESA contract (transportation and transfer to the ISS/Columbus EPF (fig.7)).

Under the assumption of both a LEO $(\sim 500$ $\mathrm{km}$ altitude) free-flyer mission or the ISS accommodation (400 $\mathrm{km}$ average altitude), the coverage of the observable atmosphere surface at the scale of $1000 \mathrm{~km}$ across and the measurement of very fast and faint phenomena like those EUSO is interested in, requires:

- Optical system with large collecting area (because of the faint fluorescence signal) and wide equivalent field of view covering a sizable half opening angle around the local Nadir (to reach geometrical factor of the order of $10^{6} \mathrm{~km}^{2} \mathrm{sr}$ ).

- Focal plane detector with high segmentation (single photon counting and high pixelization), high resolving time ( $10 \mathrm{~ns})$, contained values for weight and power.

- Trigger and read-out electronics prompt, simple, efficient, modular, capable to handle hundreds of thousands of channels, and comprehensive of a sophisticated on-board image processor acting as a trigger.

\subsection{EUSO payload: the «main telescope»}

The EUSO main telescope is presented schematically in the artistic view of fig. 8. The in-

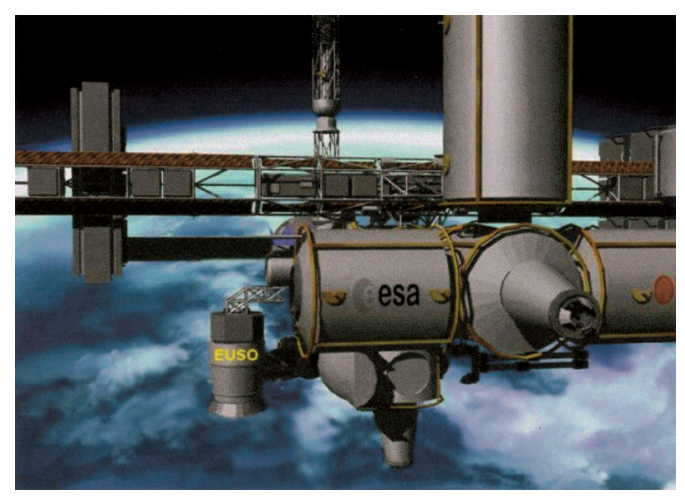

Fig. 7. EUSO at the COF-EPF.

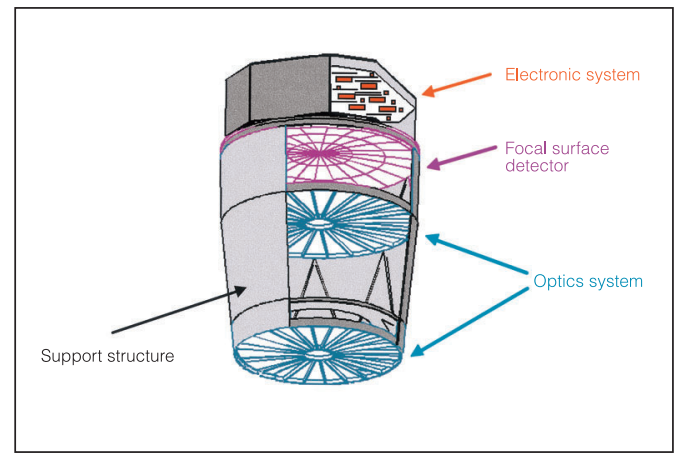

Fig. 8. View of the EUSO main telescope.

strument consists of three main parts: optics, focal surface detector, trigger and electronics system. An effective synergy between the parts constituting the instrument is of fundamental importance for achieving the EUSO scientific objectives. Optics, detector elements, system and trigger electronics have to be matched and interfaced coherently to obtain a correct response from the instrument. Scientific requirements have been of guidance for the conceptual design of the apparatus and in the choice among various possible technical solutions.

The observation from space calls for an approach different from that of the conventional ground based fluorescence experiments. For space application the instrument has to be 
compact as much as possible, highly efficient, and with a built-in modularity in its detection and electronics parts.

\subsubsection{The optics}

The optical system required for EUSO aims at finding the best compromise in the optical design, taking into account the suitability for space application in terms of weight, dimensions and resistance to the strains in launch and orbital conditions.

The optical system views a circle of radius $\sim 220 \mathrm{~km}$ on the Earth and resolves $0.8 \times 0.8 \mathrm{~km}^{2}$ ground pixels: this determines the detector size to be adopted to observe the events. The forgiving resolution requirements of EUSO suggest the consideration of unconventional solutions, identified in the Fresnel lens technology. Fresnel lenses provide large-aperture and wide-field with drastically reduced mass and absorption. The use of a broader range of optical materials (including lightweight polymers) is possible for reducing the overall weight.

The present Fresnel optical camera configuration study $\left(\mathrm{FoV} 60^{\circ}\right)$ considers two plastic Fresnel lenses with diameter $2.5 \mathrm{~m}$ and iris diaphragm $2.0 \mathrm{~m}$ diameter.

\subsubsection{The focal surface detector}

Due to the large FoV and large collecting area of the optics, the focal surface detector is constituted by several hundreds of thousands of active sensors $\left(\approx 2 \times 10^{5}\right.$ pixels $)$. The detector requirements of low power consumption, low weight, small dimension, fast response time, high quantum efficiency in UV wavelength (300-400 nm), single photoelectron sensitivity, limit the field of the possible choices to a very few devices. A suitable off-the-shelf device is the Multi-Anode Photomultiplier Hamamatsu R5900 series. These commercial photomultipliers meet closely the requirements imposed by the project. Pixel size, weight, fast time response and single photoelectron resolution are well adaptable to the EUSO focal surface detector. The organization in «macrocells» of the focal surface (a macrocell is a bi-dimensional array of $n \times n$ pixels) offers many advantages as easy planning and implementation, flexibility and redundancy. Moreover, modularity is ideal for space application. The multi-anode photomultipliers represent, in this contest, a workable solution.

\subsubsection{Trigger and electronics system}

Special attention has been given to the trigger scheme where the implementation of hardware/ firmware special functions is foreseen.

The trigger module named OUST (On-board Unit System Trigger) has been studied to provide different levels of triggers such that the physics phenomena in terms of fast, normal and slow in time-scale events can be detected. Particular emphasis has been introduced in the possibility of triggering upward showers (emerging from the earth, «neutrino candidate») by means of a dedicated trigger logic.

The FIRE (Fluorescence Image Read-out Electronics) system has been designed to obtain an effective reduction of channels and data to read-out, developing a method that reduces the number of the channels without penalizing the performance of the detection system. Rows wiredor and columns wired-or routing connections have been adopted inside every single «macrocell» ( $n \times n$ pixels unit, $\approx 100$ macrocells constitute the focal surface detector) for diminishing the number of channels to read-out.

\section{EUSO duty cycle}

The EUSO duty cycle has been estimated taking into account the following factors affecting the level of background: the ISS night time; ground locations with significant light output, natural or anthro-pomorphic; lunar cycle; clouds in the FoV strongly affecting the detection or interpretation of the EAS; ISS activities or contingencies that do not allow the operation of EUSO.

The likely EUSO duty cycle is in the range 0.1-0.15; a more precise evaluation requires a detailed assessment of the various elements, in particular of the cloud related effects: we, 
in a conservative approach, use a value of 0.1 throughout this report.

\section{Expected results}

The slope of the CR energy spectrum in the region of the GZK limit and above is poorly known because of the reduced statistics available (see fig. 1, insert). The expected counting rates for energies e.g., above $10^{20} \mathrm{eV}$, are therefore difficult to define and are strongly dependent from the assumed extrapolation for the energy spectrum. As an example, we show fig. 9 where the integral count rates are given respectively for a spectral index -2.7 and -2.3 : the counts per year above $10^{20} \mathrm{eV}$ varies from 500 to 1300 , accordingly. In the following, a spectral index of -2.7 is assumed (in a conservative way).

Figures 10 and 11 show the predicted number of EECR and neutrino events per year as a function of energy detected by EUSO in the original free-flyer and ISS configurations. Both configurations give comparable results within a small factor with the lower observational altitude of the ISS $(380 \mathrm{~km})$ counterbalancing somewhat the sensitivity afforded by the larger optics diameter of the free-flyer. The integral number of counts above an energy $E$ for the two configurations is shown in fig. 12 assuming the 2 year operational life of the free-flyer and a requested 3 year lifetime.

\subsection{Comparison with ground based observations}

In the following, a spectral index of -2.7 is assumed whenever absolute values are quoted for the counting rates above $5 \times 10^{19} \mathrm{eV}$. Currently only the AGASA and HiRes instruments are operational. The AGASA experiment near Tokyo, with coverage of about $100 \mathrm{~km}^{2}$ (about $300 \mathrm{~km}^{2} \mathrm{sr}$ ), comprises a scintillator array for electromagnetic shower particles and an array of muon detectors. The HiRes experiment in Utah consists of two fluorescence detectors at a distance of $12.6 \mathrm{~km}$ and, in a first stage, just came online. Its aperture is energy dependent and rises from $340 \mathrm{~km}^{2} \mathrm{sr}$ at $10^{19} \mathrm{eV}$ to $1000 \mathrm{~km}^{2} \mathrm{sr}$ at $10^{20} \mathrm{eV}$. The largest
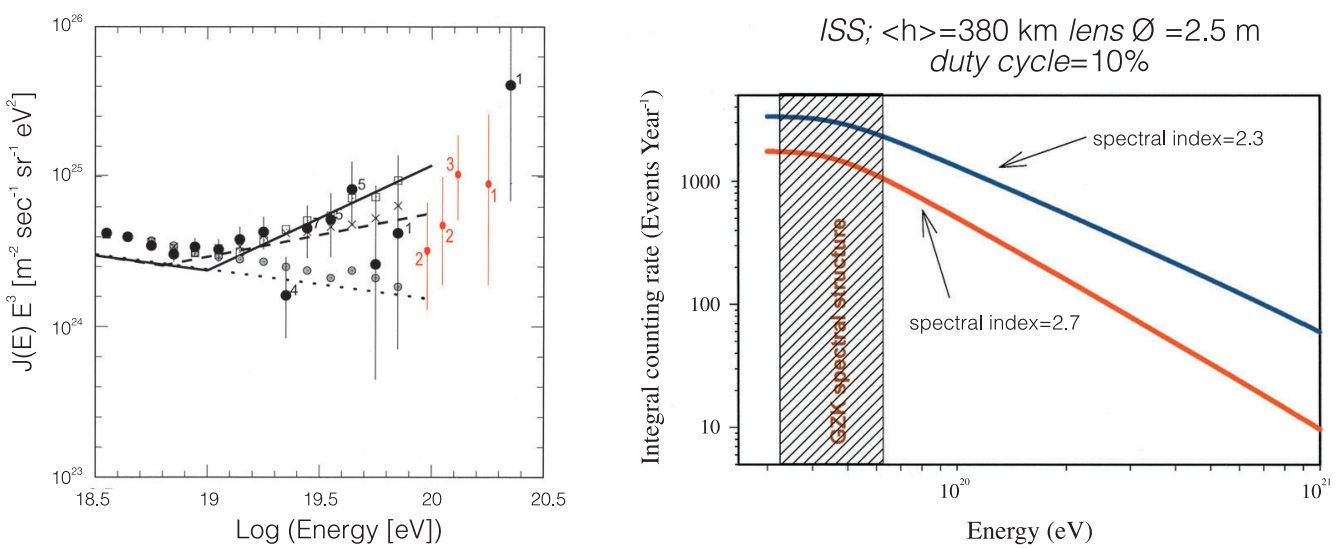

Fig. 9. (Left) This is fig. 10 from Yoshida et al. (1995): «Derived primary energy spectra expressed by eqs. (11a) (solid line) and (11b) (dashed line) and the expected values in each bin simulated under the assumptions of these spectra with the energy resolution of the present experiment (open squares and crosses). Black dots with error bars are the raw data. The case of a single power up to the highest energy is also shown by a dotted line and shaded circles.» The slope of the continuous line above $10^{19} \mathrm{eV}$ is 2.3 ; for the dashed line the slope is 2.7 . The superimposed red points are from Takeda et al. (1998). (Right) EUSO counting rates under the hypothesis of the two different spectral index assumed (see fig. 1 left). 


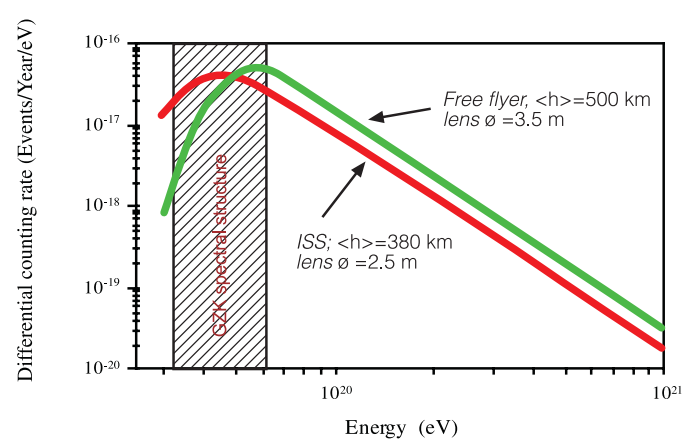

Fig. 10. Differential EECR counting rate comparison between the ISS version of the EUSO and the original free flyer. The dashed zone shows the spectral region where structure induced by the GZK cut-off is expected. The lens diameter is the maximum external diameter allowed in each configuration.

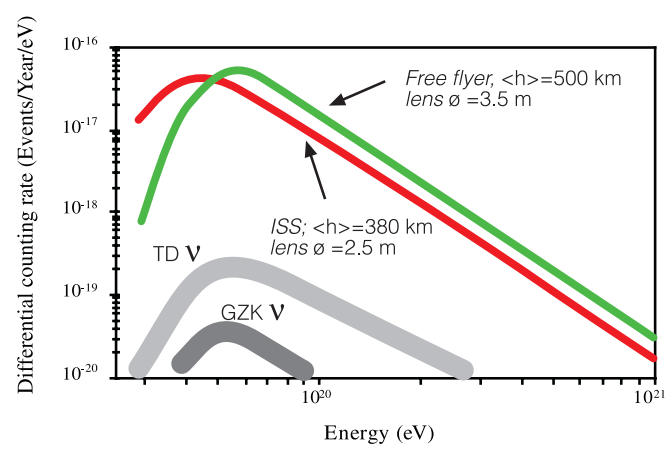

Fig. 11. The differential flux of neutrinos predicted using the topological defects model of Sigl et al. (1999) and the GZK model of Stecker et al. (1991).

planned ground-base experiment is the Pierre Auger Observatory, currently under construction in Argentina. This will consist of an array of 1600 particle detectors covering $3000 \mathrm{~km}^{2}$ and 4 fluorescence light detectors, similar to the ones used in the HiRes experiment. The hybrid detector allows cross calibration and a check of the systematic uncertainties inherent in each of the techniques. The construction is expected to be completed in 2004. By then the Auger Observatory will have an aperture of $7000 \mathrm{~km}^{2} \mathrm{sr}$, leading to about 30 events per year with energies
$>10^{20} \mathrm{eV}$. Though a second Auger Observatory is planned in the northern hemisphere, it is not clear if, or when, there will be funding for it. Auger will produce a comparable number of events to all previously observed above $10^{20} \mathrm{eV}$ in only 4 months. Nevertheless, a rate of 30 events per year is too small to follow the CR spectrum to such energies, or to obtain the detailed form of the spectrum with small statistical errors. At $10^{21}$ $\mathrm{eV}$ only about 5 events are expected in 10 years of operation. At least one order of magnitude more statistics is desirable. By the year 2006, provided Auger is completed on schedule, the world data set will comprise about 100 events above $10^{20} \mathrm{eV}$ and perhaps one or two events above $10^{21} \mathrm{eV}$, if the spectrum continues without a GZK cut-off. The existence or non-existence of the GZK cut-off will most likely be established by then. However, a definite answer concerning the origin and possible identification of sources will certainly rely on the precise spectral form and on the arrival direction distribution. In both cases, good statistics are vital to distinguish between different competing scenarios.

For EUSO, an effective geometrical factor of $5 \times 10^{5} \mathrm{~km}^{2} \mathrm{sr}$ (fig. 13) and an observing efficiency of 0.1 gives an expected event rate of $\sim 500$ per year for EECR with energies $\geq 10^{20} \mathrm{eV}$ (no GZK suppression and spectral index of 2.7), or some hundreds per year with GZK suppression. The

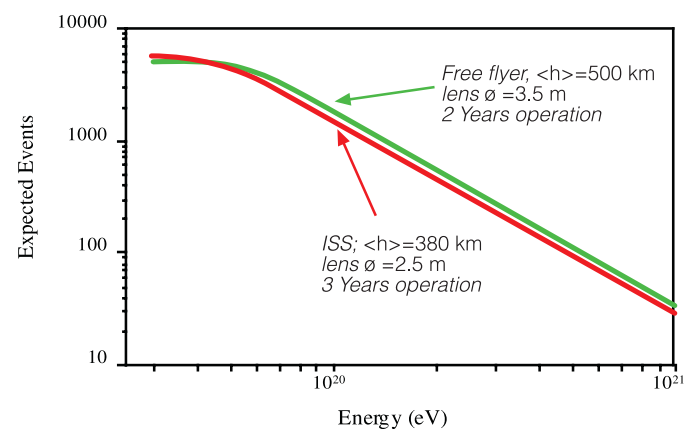

Fig. 12. The integral count rates above an energy $E$ predicted for the original free flyer proposal with 2 years of operations and the ISS configuration with 3 years operations. 


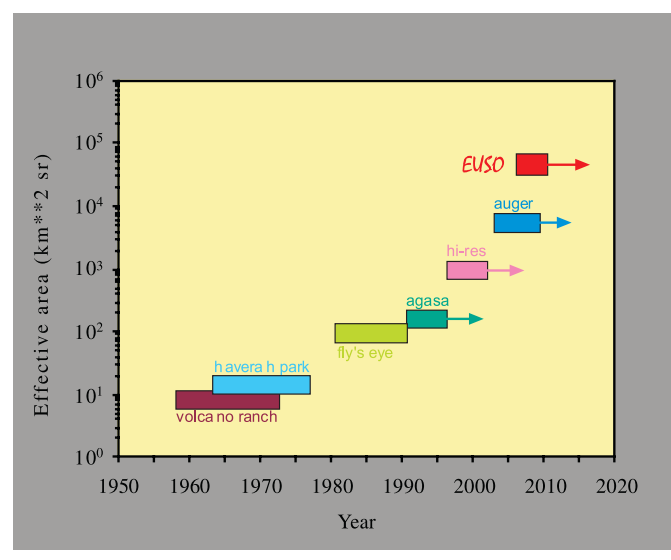

Fig. 13. A comparison of the EUSO effective area with ground based facilities.

total number of events per year with the lowenergy threshold of $>3 \times 10^{19} \mathrm{eV}$ is $>1700$. For EECR neutrinos the expected rates vary from a few per year (GZK processes, AGN, GRB sources, etc.) to $\sim 150$ per year if top-down processes dominate.

\section{Acknowledgements}

A general acknowledgemt is due to the EUSO collaboration. I am deeply indebted to M.C. Maccarone for her determinant contribution.

\section{REFERENCES}

ESA/ESTEC (2000): EUSO Accommodation Study Report, ESA/MSM-GU/2000.462/AP/RDA, December 2000.

SCARSI, L. et al. (2000): EUSO-Extreme Universe Space Observatory, Proposal for the ESA F2/F3 Missions, January 2000 (http://www.ifcai.pa.cnr.it/ EUSO/docs/ EUSOproposal.pdf).

Sigl, G., S. Lee, P. Bhattacharjee and S. Yoshida (1999): Probing grand unified theories with cosmicray, gamma-ray, and neutrino astrophysics, Phys. Rev. $D, \mathbf{5 9}, 043504$, pp. 8.

STECKER, F.W. and M.H. SALAMON (1996): High-energy neutrinos from quasars, Space Sci. Rev., 75, 341-355.

SteCKER, F.W., C. DONE, M.H. SAlamon and P. SOMmers (1991): High-energy neutrinos from active galactic nuclei, Phys. Rev. Lett., 66 (21), 2697-2700.

Takeda, M., N. Hayashida, K. Honda, N. Inoue, K. KaDOTA, F. KAKIMOTO, K. KaMATA, S. KaWAGUCHI, Y. Kawasaki, N. Kawasumi, H. Kitamura, E. Kusano, Y. Matsubara, K. MuraKami, M. Nagano, D. NishiKaWA, H. OHOKA, N. SAKAKI, M. SASAKI, K. SHINOZAKI, N. SOUMA, M. TeSHIMA, R. TORII, I. TSUSHIMA, Y. UCHIHORI, T. YAMAMOTO, S. YOSHIDA and H. YoshiI (1998): Extension of the cosmic-ray spectrum beyond the predicted Greisen-Zatsepin-Kuz'min cut-off, Phys. Rev. Lett., 81 (6), 1163-1166.

Yoshida, S., N. Hayashida, K. Honda, M. Honda, S. Imaizumi, N. InOUE, K. Kadota, F. KaKimoto, K. Kamata, S. Kawaguchi, N. Kawasumi, Y. Matsubara, K. MuraKami, M. NAGANO, H. OHOKA, M. Teshima, I. Tsushima and H. YoshiI (1995): The cosmic ray energy spectrum above $3 \times 10^{18} \mathrm{eV}$ measured by the Akeno Giant Air Shower Array, Astroparticle Phys., 3 (2), 105-123.

YoshiDA, S., G. SigL and S. LeE (1998): Extremely high energy neutrinos, neutrino hot dark matter, and the highest energy cosmic rays, Phys. Rev. Lett., 81 (25), 5505-5508.

WAXMAN, E. and J. BAHCALL (1997): High energy neutrinos from cosmological gamma-ray burst fireballs, Phys. Rev. Lett., 78 (12), 2292-2295. 